\title{
STRONGLY CORRELATED ELECTRONS IN A MAGNETIC FIELD: FERMI LIQUID VERSUS SPIN LIQUID
}

\author{
J. SPALIEK \\ Institute of Theoretical Physics, Warsaw University, Hoża 69, 00-681 Warszawa, Poland \\ AND W. WójCIK \\ Institute of Physics, Technical University, Podchorążych 1, 30-084 Kraków, Poland
}

\begin{abstract}
We compare the Fermi liquid (FL) and statistical spin liquid (SSL) representations of the electronic properties for strongly correlated systems. In particular, we discuss the entropy, as well as the magnetization as a function of band filling, temperature and magnetic field. These properties are quite different in the two (FL, SSL) states. Antiferromagnetic state disappears for a small number of holes in the doped Mott insulator.
\end{abstract}

PACS numbers: 71.28.+d, 75.10.Lp

\section{Statistical spin liquid}

The electron liquid close to (but below) the Mott insulating limit is commonly represented by an almost localized Fermi liquid [1] of electrons in a half-filled band. In such a Fermi liquid (FL) the effective mass of quasiparticles is adjusted self-consistently from the balance between the renormalized band and the Coulomb parts of the total electronic energy, and diverges at the localization boundary [2]. In the presence of applied magnetic field the quasiparticle mass depends strongly on its spin direction [3] and becomes large for the spin minority carriers when the magnetization approaches saturation. However, there is one fundamental problem with the concept of heavy quasiparticles in the Mott insulating limit. Namely, in FL state the configurational entropy per carrier in the high-temperature limit is $2 k_{\mathrm{B}} \ln 2, \operatorname{not} k_{\mathrm{B}} \ln 2$, as required for localized magnetic moments. This difficulty is overcome by defining a first-order Fermi-liquid-localized-moment phase boundary, i.e. by treating the Fermi liquid and the localized-moment states as separate phases in a thermodynamic sense [2]. The localization boundary is intuitively appealing in the case of half-filled band when a metal transforms with growing correlations into a Heisenberg magnet. Nonetheless, a question remains about the nature of metallic state in a nonhalf-filled band situation in the strong-correlation limit, when no correlated metal-magnetic insulator transition occurs. 
To describe a strongly correlated metal a novel state called the statistical spin liquid (SSL) was invoked [4-6]. This liquid is characterized by absence of doubly occupied opposite-spin configurations in reciprocal $(k)$ space. The projection of $\{|k \uparrow \downarrow\rangle\}$ states reflects a grouping of single-particle states into two Hubbard subbands [7], each containing nearly the same number of available $k$ states for an almost half-filled band situation. A proper configurational entropy is retrieved automatically as SSL evolves into localized spins (i.e. band filling $n \rightarrow 1$ ). The SSL concept has received a substantial impulse after the role of doubly occupied $k$ state configuration exclusion in two-dimensional systems has been emphasized [8,9].

In this communication we report briefly on magnetic properties for SSL and compare them with those for FL state. In a separate note [10] we summarize the corresponding mean-field results for a superconducting phase in two space dimensions.

\section{Magnetic properties of statistical spin liquid}

We start from the effective Hubbard model in the strong correlation limit [11] in which doubly occupied configurations have been projected out in real space. It has the form.

$$
H=\sum_{i j \sigma}^{\prime} t_{i j} b_{i \sigma}^{\dagger} b_{j \sigma}+\sum_{i j}^{\prime} \frac{2 t_{i j}^{2}}{U}\left(S_{i} \cdot S_{j}-\frac{1}{4} \nu_{i} \nu_{j}\right),
$$

in which the projected fermion operators, e.g. $b_{i \sigma} \equiv a_{i \sigma}\left(1-n_{i-\sigma}\right)$, represent single-particle hopping, as expressed by the first term, whereas the second term represents kinetic exchange interactions among correlated electrons. Other symbols are standard [12]. Next, we transform (1) to reciprocal $(k)$ space the double occupancies again. This leads to the explicit form of the Hamiltonian which in the interesting us case with a spin-density wave state reduces to the following form in the mean-field approximation:

$$
\begin{aligned}
& H=\sum_{k_{\sigma}}\left(\varepsilon_{k^{b}}{ }^{\dagger}{ }_{k_{\sigma}}{ }^{b} k_{\sigma}+\varepsilon_{k+} Q^{b_{k+}^{\dagger}} \boldsymbol{Q}_{\sigma}{ }^{b} k_{+} Q_{\sigma}\right) \\
& +\frac{1}{2} J(Q)\left\langle S^{z}\right\rangle \sum_{k_{\sigma}} \sigma\left(b_{k_{+}+Q_{\sigma}}^{\dagger} b_{k_{\sigma}}+b_{k_{\sigma}}^{\dagger} b_{k+} Q_{\sigma}\right)-J(Q)\left\langle S^{z}\right\rangle^{2} N
\end{aligned}
$$

In this equation the new projected fermion operators are $b_{k_{\sigma}} \equiv a_{k_{\sigma}}\left(1-n_{k-\sigma}\right)$,

$$
J(Q)=\left(\frac{4}{U}\right) \sum_{j(i)} t_{i j}^{2} \exp \left(\mathrm{i} \boldsymbol{Q} \cdot\left(\boldsymbol{R}_{j}-\boldsymbol{R}_{i}\right)\right)
$$

and $\boldsymbol{Q}$ is the wave vector of a longitudinal spin-density wave, and

$$
\left\langle S^{z}\right\rangle=\frac{1}{2 N} \sum_{k}\left(\left\langle b_{k+}^{\dagger} Q_{\uparrow} b_{k \uparrow}-b_{k+}^{\dagger} Q_{\downarrow} b_{k \downarrow}\right\rangle\right)
$$

is the magnetic moment magnitude in the ordered state. The summation over $k$ is performed for the vectors in the reduced Brillouin zone created by the magnetic superstructure. The Hamiltonian (2) is used to determine the anticommutator Green 
functions $\left\langle\left\langle b_{k_{\sigma}} \mid b_{k_{\sigma}}^{\dagger}\right\rangle\right\rangle$ and $\left\langle\left\langle b_{k+Q_{\sigma}} \mid b_{k_{\sigma}}^{\dagger}\right\rangle\right\rangle$, which are calculated in the mean-field approximation for the first two moments [13]. Such procedure leads to the $2 \times 2$ matrix form of the Green function

which is

$$
G_{\boldsymbol{k}_{\sigma}}(\omega) \equiv\left(\begin{array}{cc}
\left\langle\left\langle b_{\boldsymbol{k}_{\sigma}} \mid b_{\boldsymbol{k}_{\sigma}}^{\dagger}\right\rangle,\right. & \left\langle\left\langle b_{\boldsymbol{k}_{\sigma}} \mid b_{\boldsymbol{k}_{+} \boldsymbol{Q}_{\sigma}}^{\dagger}\right\rangle\right\rangle \\
\left\langle\left\langle b_{\boldsymbol{k}_{+} \boldsymbol{Q}_{\sigma}} \mid b_{\boldsymbol{k}_{\sigma}}^{\dagger}\right\rangle\right\rangle, & \left\langle\left\langle b_{\boldsymbol{k}_{+} \boldsymbol{Q}_{\sigma}} \mid b_{\boldsymbol{k}_{+} \boldsymbol{Q}_{\sigma}}^{\dagger}\right\rangle\right.
\end{array}\right)
$$

$$
\begin{aligned}
& G_{k_{\sigma}}(\omega)=\frac{1}{2 \pi\left(\omega-\omega_{1 k}\right)\left(\omega-\omega_{2 k}\right)} \\
& \quad \times\left(\begin{array}{cc}
-\varepsilon_{k+} \boldsymbol{Q}-\mu+\omega, & \sigma \Delta\left(1-n_{\boldsymbol{k}+\boldsymbol{Q}-\sigma}\right) \\
\sigma \Delta\left(1-n_{\boldsymbol{k}_{-\sigma}}\right), & -\varepsilon_{\boldsymbol{k}}-\mu+\omega
\end{array}\right),
\end{aligned}
$$

where $\mu$ is the chemical potential, and $\Delta=J(Q)\left\langle S^{z}\right\rangle$ is the Slater gap in SSL state. The Green function matrix allows for determination of the correlation functions $\left\langle b_{k_{+}}^{\dagger} Q_{\sigma}{ }^{b} k_{\sigma}\right\rangle$ and $\left\langle b_{k_{\sigma}}^{\dagger}{ }^{b} k_{\sigma}\right\rangle \equiv n_{k_{\sigma}}$ which in turn determine self-consistent equations for $\left\langle S^{z}\right\rangle$ and $\mu$. The quasiparticle energies are

$$
\begin{aligned}
\omega_{1,2 k} & =\left(\frac{\varepsilon_{k}+\varepsilon_{k+Q}}{2}\right) . \\
& \pm \sqrt{\left(\frac{\varepsilon_{k}-\varepsilon_{k+Q}}{2}\right)^{2}+J(Q)^{2}\left(1-n_{k-\sigma}\right)\left(1-n_{k+Q-\sigma}\right)-\mu .}
\end{aligned}
$$

We have solved the equations for $\left\langle S^{z}\right\rangle$ and $\mu$ for the case of antiferromagnetic ordering on square lattice for which $Q=(\pi / a)(1,1)$, and $a$ is lattice constant. For that situation perfect nesting condition $\varepsilon_{k+Q}=-\varepsilon_{k}$ is met. In Fig. 1 we display our results for the order parameter $\left\langle S^{z}\right\rangle$ as a function of hole number $\delta=1-n$; the value of the exchange constant $J=4 t^{2} / U$ was taken $2000 \mathrm{~K}$ to reflect there situation for $\mathrm{CuO}_{2}$ plane of a parent antiferromagnetic material for the high temperature superconductor. The ordering takes place only in the regime of $\delta$ very close to half-filling. This type of rapid destruction of antiferromagnetic order is indeed observed in $\mathrm{La}_{2-x} \mathrm{Sr}_{x} \mathrm{CuO}_{4}$ systems [14]. No attempt was made as yet to obtain a solution for an arbitrary $\boldsymbol{Q}$. In the inset we display the normal-state entropy per site as a function of band filling $n$; only in the SSL case a proper Mott insulating value $k_{\mathrm{B}} \ln 2$ per particle is obtained. To characterize the magnetic properties in the normal state we calculated the magnetization curves shown in Fig. 2, for both SSL and FL states, this time for constant shape of the density of states. SSL magnetizes much easier than FL; this is because no doubly occupied (spin singlet) configurations are encountered in the former case. Additionally, in the inset to Fig. 2 we exhibit the static magnetic susceptibility which has the Curie-Weiss form. The temperature dependence is weak because the antiferromagnetic interactions are very strong $\left(k_{\mathrm{B}} T<J\right)$. The susceptibility in the $\mathrm{FL}$ case is constant, since the density of states curve is featureless. 


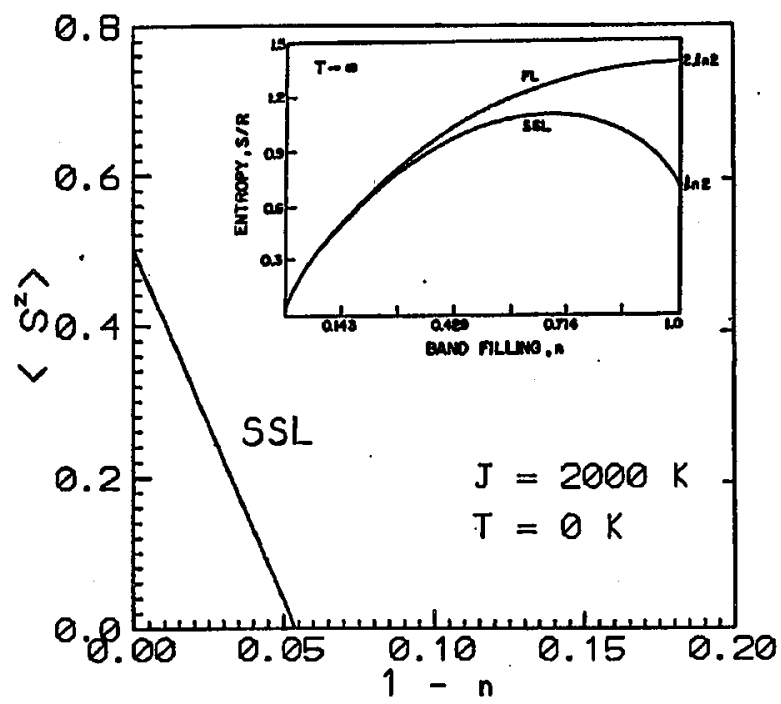

Fig. 1. The antiferromagnetic order parameter $\left\langle S^{z}\right\rangle$ as a function of hole number $\delta=1-n$ for a square lattice and exchange integral $J=2000 \mathrm{~K}$. The inset displays the band filling dependence of the normal state entropy (in gas constant units) for statistical spin liquid (SSL) and Fermi liquid (FL) states.

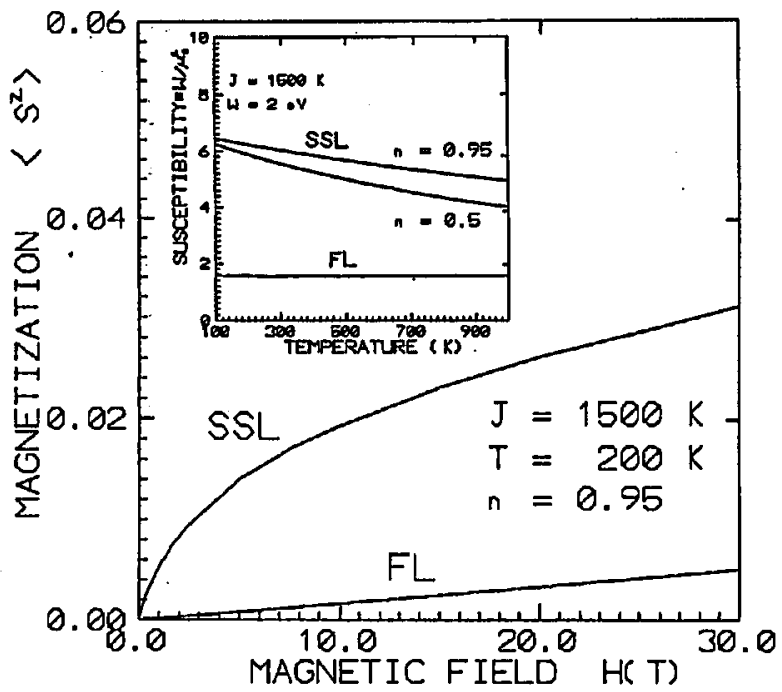

Fig. 2. Magnetization curve for SSL and FL cases as a function of applied magnetic field. The inset shows a weak Curie-Weiss dependence for SSL, and the Pauli paramagnetism for FL; a featureless form of the density of states was taken in both cases. 


\section{Concluding remarks}

The present formulation relies on a novel statistics $[4-6,8]$ of strongly correlated electrons, which was applied to the case of a square lattice with strong in-plane antiferromagnetic interactions. We believe that this novel statistics describes some principal nontrivial properties of the two-dimensional magnetic and superconducting materials.

The work was supported by the grants of the Committee for Scientific Research.

\section{References}

[1] For recent review see: J. Spałek, J. Solid State Chem. 88, 70 (1990).

[2] J. Spałek, A. Datta, J.M. Honig, Phys. Rev. Lett. 59, 728 (1987).

[3] J. Spałek, P. Gopalan, Phys. Rev. Lett. 64, 2823 (1990).

[4] J. Spałek, W. Wójcik, Phys. Rev. B 37, 1532 (1988).

[5] J. Spałek, W. Wójcik, in: Sci. Bull. Jagellonian University 29, 87 (1990).

[6] J. Spałek, Phys. Rev. B 40, 5180 (1989); Physica B 163, 621 (1990).

[7] J. Hubbard, Proc. R. Soc. Lond. A 281, 401 (1964).

[8] P.W. Anderson, Phys. Rev. Lett. 64, 2823 (1990); 65, 2306 (1990).

[9] P.W. Anderson, unpublished lecture notes.

[10] K. Byczuk, J. Spałek, Acta Phys. Pol. A 85, ... (1994).

[11] K.A. Chao, J. Spałek, A.M. Oleś, J. Phys. C 10, L271 (1977).

[12] J. Spałek, Phys. Rev. B 38, 533 (1988).

[13] L. Roth, Phys. Rev. 184, 451 (1969).

[14] Y. Kitaoka, K. Ishida, T. Kobayashi, K. Amaya, K. Asayama, Physica C 153-155, 733 (1988). 